\title{
Asbestos related pleuropulmonary lesions and the erythrocyte sedimentation rate
}

\author{
GUNNAR HILLERDAL \\ From the Department of Lung Medicine, University Hospital, Uppsala, Sweden
}

\begin{abstract}
Asbestos related lesions of the lung parenchyma and the pleura can be divided into three main types: parietal pleural plaques, diffuse interstitial fibrosis ("classical asbestosis"), and a third type of reaction affecting both the pleura and the lung parenchyma. The last type includes such lesions as acute pleurisy, diffuse pleural thickening, and rounded atelectasis. Among 1344 patients with asbestos related pleural lesions, 1190 had pleural plaques, 29 of whom also had pulmonary fibrosis (asbestosis); 83 had unilateral sequelae of pleurisy, of whom nine had asbestosis; and 71 had bilateral sequelae of pleurisy, of whom 23 also suffered from asbestosis. The erythrocyte sedimentation rate (ESR) was measured in 184 patients -79 with pleural plaques, 44 with unilateral sequelae of pleurisy, and 61 with bilateral sequelae. In patients with pleural plaques with or without asbestosis the ESR was usually normal, the mean being $9.6 \mathrm{~mm}$ in one hour. Among patients with sequelae of pleurisy, however, many had a raised ESR. The mean ESR was $25.7 \mathrm{~mm}$ in one hour in those with bilateral changes and 13.2 in those with unilateral changes. Statistical analysis showed that in this group of subjects the presence of sequelae of pleurisy was a highly significant determining factor for the ESR $(p<0.0001)$. Asbestosis alone was not a significant determining factor but there was a low grade of significance for the combined effect of asbestosis and sequelae of pleurisy $(p<0.05)$. These findings suggest that the pathogenesis of the various changes is different.
\end{abstract}

Pleural and parenchymal lung lesions are commonly found after exposure to asbestos. Pleural plaques are the best known form but more extensive asbestos related pleural changes also occur. The pleuropulmonary changes related to previous asbestos exposure can be divided into three distinctive types: classical asbestosis (diffuse fibrosis of the lung parenchyma); pleural plaques, which are limited to the parietal pleura; and other reactions, which include so called diffuse pleural thickening, acute pleurisy, and rounded atelectasis. Experience with more than 1000 patients suffering from asbestos related disease followed for more than 20 years both prospectively and retrospectively suggests that the last group probably represents variations on the same theme-namely an acute pleurisy or the remnants thereof. The close relationship between the different forms in this last group can be demonstrated by computed tomography.

Address for reprint requests: Dr G Hillerdal, Department of Lung Medicine, University of Uppsala, University Hospital, 750 14 Uppsala, Sweden.

Accepted 8 May 1984
We have noted that occasional patients have a persistent but unexplained increase in their erythrocyte sedimentation rate (ESR). Since these patients seemed to be mainly of the third type referred to above we thought that this warranted further investigation. Routine blood tests were otherwise only rarely performed since the results were almost invariably normal in persons with pleural plaques only.

\section{Methods}

STUDY POPULATION

In the county of Uppsala, Sweden, with a population of about 250000 , a general health survey has been in progress since the early 'sixties. The survey includes a miniature chest radiograph. Adults are invited to participate every second or third year, and $\stackrel{D}{\stackrel{D}{~}}$ the overall participation rate is $70 \% .^{.}$In the early $\stackrel{\mathcal{Q}}{\rightarrow}$ 'seventies there was a noticeable increase in pleural 0 changes due to asbestos, and this has since been studied prospectively. ${ }^{2}$

All people with features suggestive of pleural plaques on the miniature chest radiograph are recalled 752 
for a full size chest radiograph (posteroanterior and lateral views) and questioned about exposure to asbestos and smoking history, and a clinical history is obtained. They are advised to stop smoking and offered a further examination every second year or more often if this seems warranted. In the early years only patients with typical pleural plaques were included in the study and changes such as a blunted costophrenic angle led to exclusion. Some people with "pure" plaques were, however, found at a later examination to have developed changes that would earlier have led to their exclusion. In other patients with blunted costophrenic angles it was found that at earlier examinations they had only typical pleural plaques. A prerequisite for inclusion was the presence of bilateral changes. In addition to those identified during the health survey, a few subjects were discovered by other means or referred directly to the clinic.

\section{DEFINITIONS}

Pleural plaques These were defined as sharply limited thickenings of the parietal pleura situated either on the chest wall or on the diaphragm. The width of tangentially observed plaques should be at least $5 \mathrm{~mm}$ (grade b according to the ILO scheme); if the plaques were clearly visible face on in the frontal view they were considered to be at least $5 \mathrm{~mm}$ thick. If plaques were seen only on the diaphragm they had to be calcified to be accepted. Further criteria were the presence of bilateral lesions and absence of blunting of the costophrenic angles.

Sequelae of pleurisy These were diagnosed whenever changes of the following types were seen, alone or in combination: (a) Blunted costophrenic angle or angles. (b) Diffuse pleural thickening, not sharply delimited, covering at least half of the chest wall. This is not the same as large pleural plaques. The thickening can be extensive and affect the whole lung. The corresponding costophrenic angle is almost invariably blunted. (c) "Crow's feet." This term, coined by Mackenzie and Harries, ${ }^{3}$ is a descriptive term for fibrous strands extending from a thickened pleura, sometimes converging to a "shrinking centre," which is often best seen in the lateral view (figs 1-3). These strands are fibrous bundles that are mainly situated within the lung parenchyma and can traverse the whole lung; they are strikingly demonstrated on computed tomography scanning (figs $1(c)$ and $2(c)$ ). The visceral pleura is sometimes considerably thickened and the strands may extend from diffuse pleural thickening. In most cases the costophrenic angle is blunted. (d) Rounded atelectasis: a tumour like atelectasis of part of the peripheral lung, which has been "caught" in some of the "crow's feet" causing obliteration of some small bronchi. ${ }^{4-6}$

Pulmonary asbestosis This category included all patients with some grade of dyspnoea, static lung volumes of less than $80 \%$ of predicted normal value, and basal crackles, with radiological lesions of some of the types described above. Thus all patients with pulmonary asbestosis also had pleural changes.

Acute asbestos pleurisy This was diagnosed in the way now generally accepted with three criteria: exclusion of other causes; exposure to asbestos; and follow up of at least three years to exclude malignancy. Subjects were divided into three groups according to the pleural changes: (1) pleural plaques alone; (2) unilateral sequelae of pleurisy and plaques on the other side; (3) bilateral sequelae of pleurisy. Each of these groups was further subdivided into those with and those without asbestosis, making six groups in all. The radiological classification was made on the basis of the chest radiographic appearances; information from computed tomography was not used for this purpose.

Laboratory tests During two years (1981 and 1982) blood tests were performed on all patients with asbestosis or sequelae of pleurisy or both who came for their regular check up and for one month on all patients attending with pleural plaques alone. Patients with diseases known to affect the ESR (for example, rheumatoid arthritis, acute infections, etc) were excluded. In some cases serial blood tests were available over many years, mainly in patients in whom a high ESR had not returned to normal after acute pleurisy. In a few patients electrophoresis of serum proteins and tests for rheumatoid and antinuclear factors were also performed. This was again mainly in those who had a raised ESR.

\section{STATISTICAL ANALYSIS}

For the statistical evaluation we used analysis of variance for unbalanced data of two way design with interaction. $^{7-9}$

\section{Results}

At the end of year 19821344 people had been registered (table 1). In 1045 subjects $(77 \cdot 8 \%$ ) serial radiographs covering more than 15 years were available. The vast majority of these were men above the age of 40 . In 184 cases blood tests had been performed. The mean ESR was within normal limits in patients who had pleural plaques alone. In striking contrast, those with bilateral sequelae of 


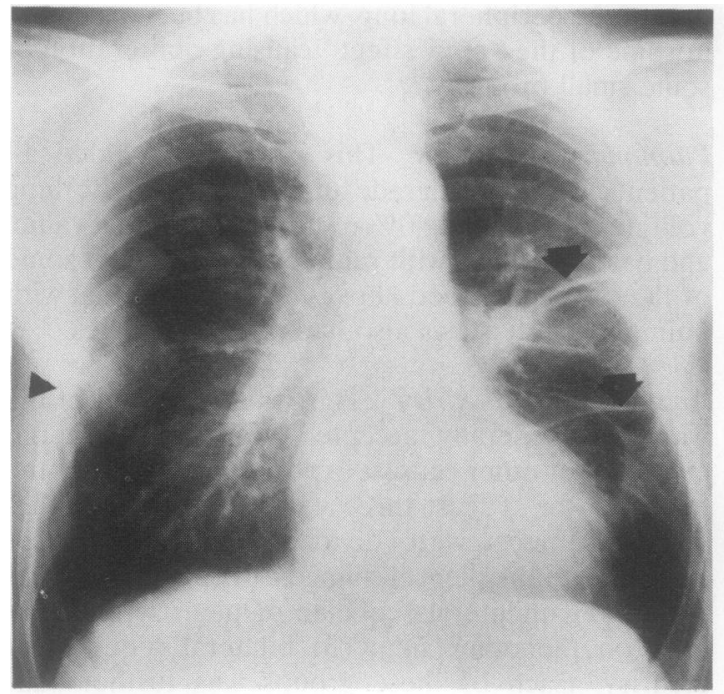

$A$

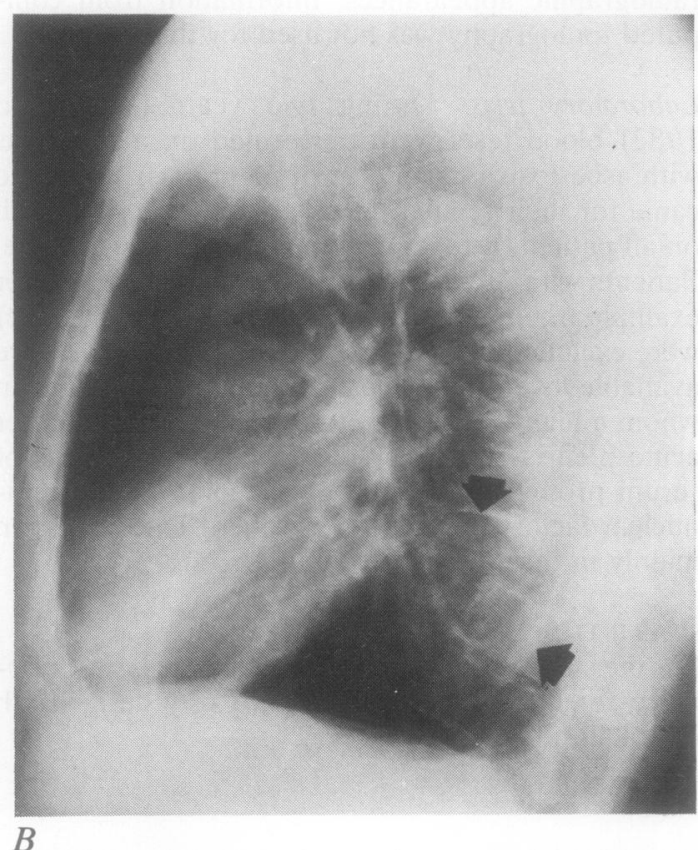

Fig 1 Unilateral sequelae of pleurisy. $A$-anteroposterior view; $B$-lateral view. On the right side there are well developed plaques (arrowhead) and on the left "crows' feet" (broad arrows). The "crows' feet" are also visible on the lateral view (broad arrows). On computed tomography scan (C and D) plaques (arrowheads) are visible on the right side. Note the sharp demarcation from the normal lung parenchyma, which differs from the pleural thickening with fibrous strands passing into the lung on the left side (broad arrows). These are the "crows' feet." In C a pseudotumour is visible posteriorly on the left. Close study shows that the pleura adjacent to the vertebra on the right is also thickened. Small fibrous strands extend into the lung. Thus the computed tomography scan shows bilateral sequelae of pleurisy.
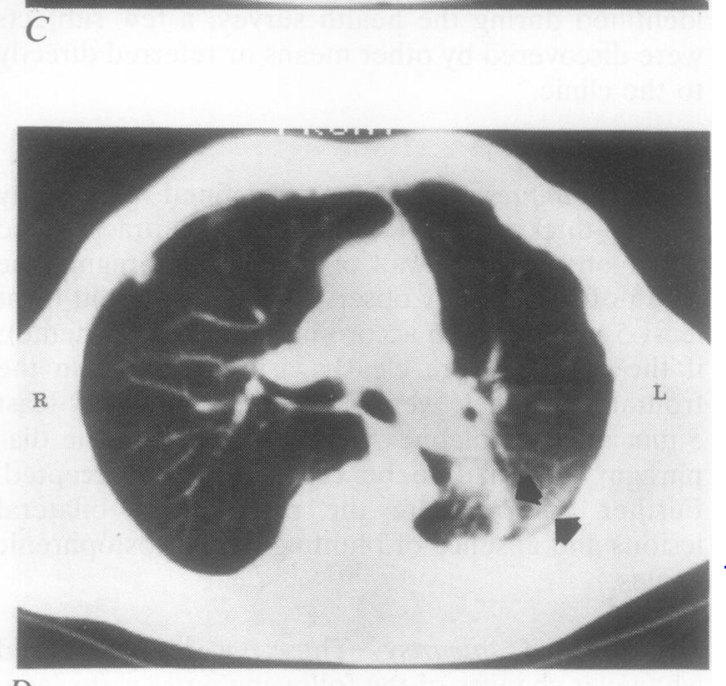

D

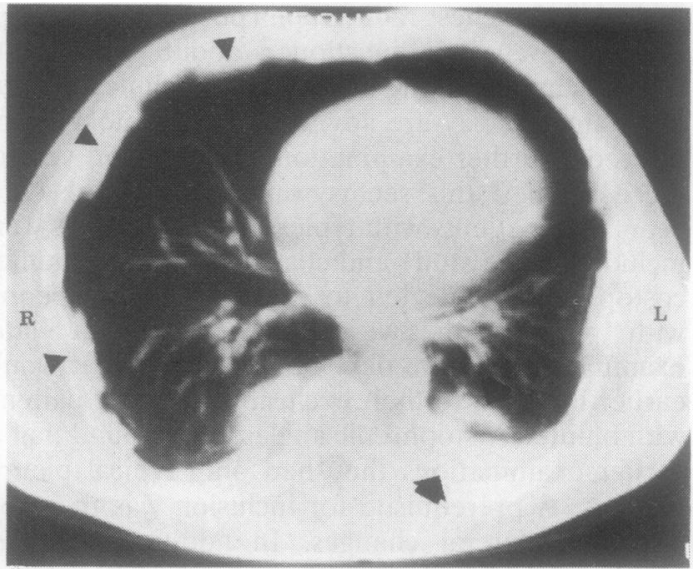




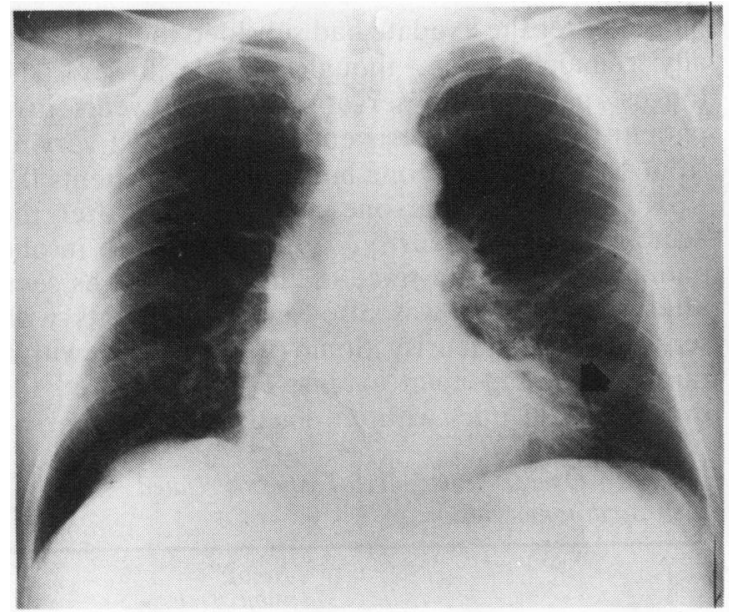

A

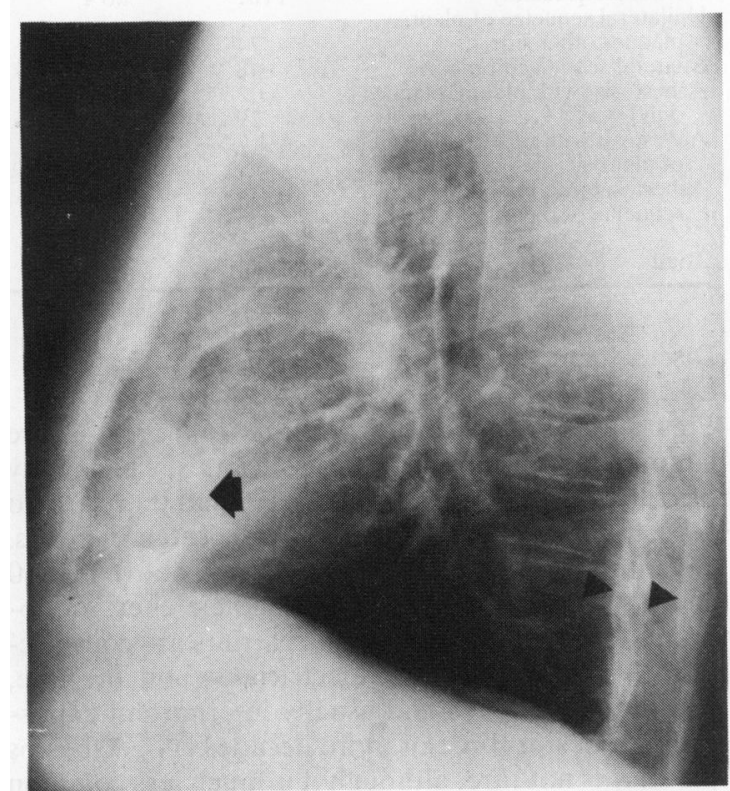

$B$

pleurisy showed a considerable rise in ESR (table 2). Among persons with pleural plaques but no asbestosis $80 \%$ had an ESR of $10 \mathrm{~mm}$ in one hour or less; whereas among those with bilateral sequelae of pleurisy with and without asbestosis the corresponding figures were respectively $15 \%$ and $24 \%$ (fig 4). Statistical analysis showed that sequelae of pleurisy was a very highly significant determining factor for the ESR ( $p<0.0001)$ in this group of subjects; the occurrence of asbestosis alone was not a significant determining factor but there was a low

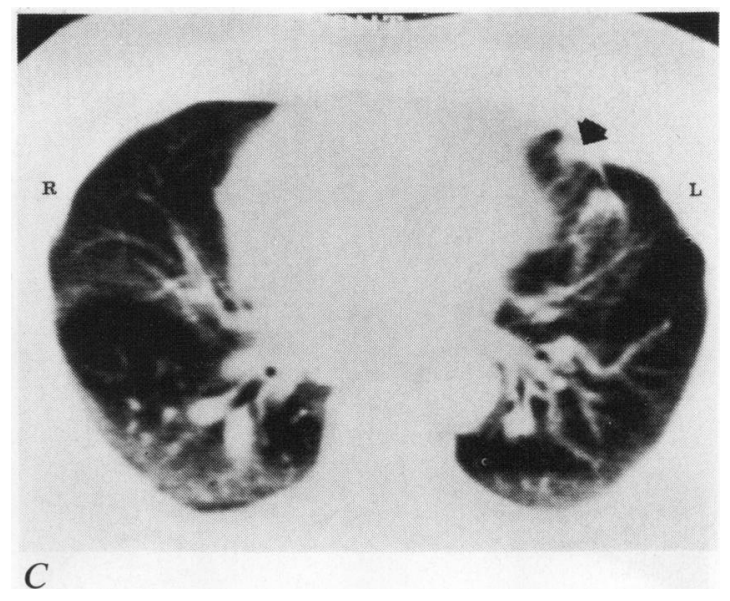

Fig 2 Right sided plaques, left sided sequelae of pleurisy. $A$-anteroposterior view. Appearances of "pseudotumour" are visible near the heart (broad arrow). B-lateral view. The pseudotumour is situated anteriorly. Plaques (arrowheads) are visible on both sides posteriorly. C-computed tomography scan.

grade of significance for the combined effect of asbestosis and sequelae of pleuritis $(p<0.05)$.

Serum protein electrophoresis was performed in 41 cases. The immunoglobulin concentrations were normal or slightly raised; a polyclonal increase was seen in six of 19 patients with sequelae of pleurisy (32\%) and in seven of 26 in the other groups (27\%). In addition, some patients showed non-specific features of immunological activity (that is, increased haptoglobin concentrations).

Four of 30 tests for rheumatoid factor and three 

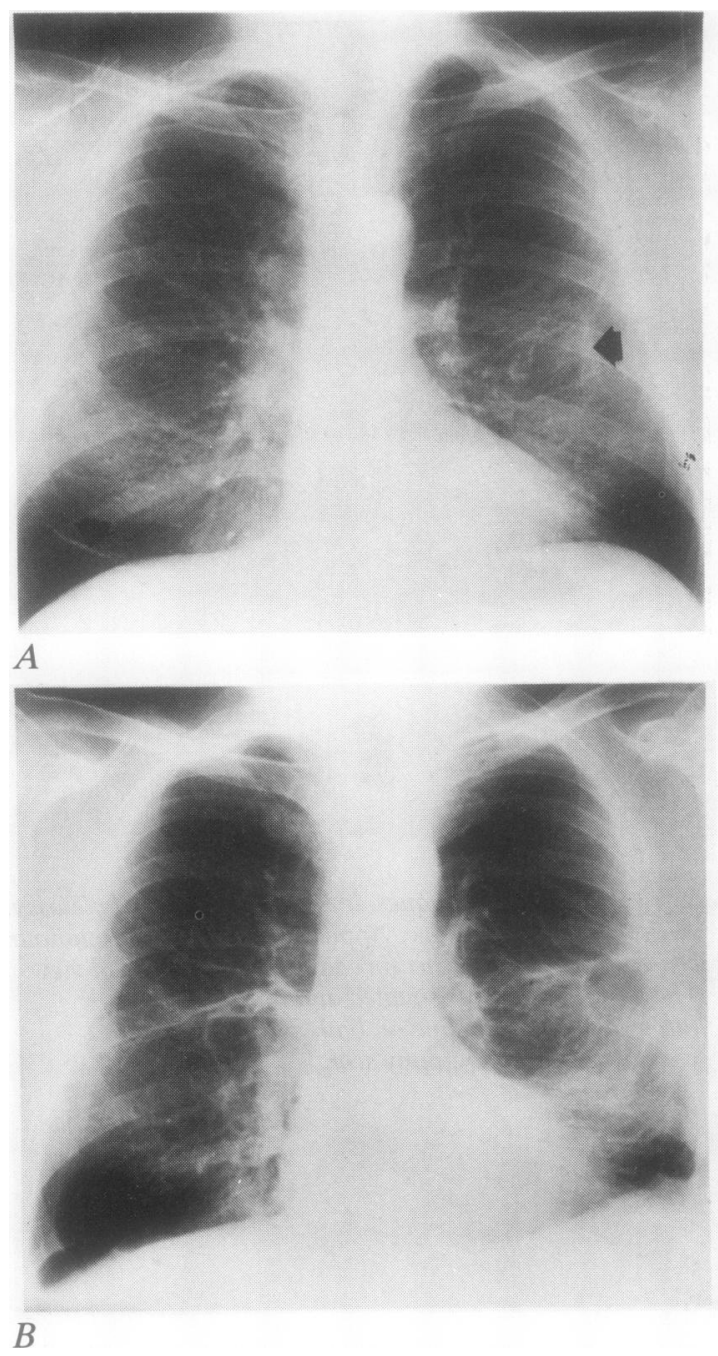

Fig 3 Development of pleural lesions. A-appearances in 1978. There are discrete changes of the "crows' feet" type on the left side and near the diaphragm on the right (broad arrows). B-appearances in 1982. There has been progression on both sides, with the development of bilateral pleural thickening further crows' feet and blunting of the costophrenic angles.

of 29 tests for antinuclear factor gave a weakly positive result. All three results positive for antinuclear factor came from the group of 10 patients with bilateral sequelae of pleurisy. Results positive for rheumatoid factor were found in all groups.

Twenty patients were seen during an attack of pleurisy and then followed up for some years. There were 13 patients with unilateral and seven with bilateral pleural exudate. The ESR varied from 6 to $85 \mathrm{~mm}$ in one hour, with a mean of 39 in the acute phase. After the exudate had subsided the ESR usually remained high, though a little lower. The longest period of observation was 13 years in a patient who had a persistently raised ESR, varying from 15 to $50 \mathrm{~mm}$ in one hour. In some patients the ESR became normal one or two years after the acute episode of pleurisy. This was the case in one patient in whom no trace of the pleurisy was seen afterwards, but it was also seen in patients with sequelae of the pleurisy. Some of the patients with a raised ESR had been extensively investigated for this in other clinics without positive results.

Table 1 Overall prevalence of asbestos related pleuropulmonary lesions

\begin{tabular}{lcr}
\hline & $\begin{array}{l}\text { No of } \\
\text { subjects }\end{array}$ & \multicolumn{1}{l}{$\%$} \\
\hline Pleural plaques only & 1161 & $86 \cdot 4$ \\
$\begin{array}{l}\text { Unilateral sequelae of pleurisy, } \\
\text { plaques other side }\end{array}$ & 74 & $5 \cdot 5$ \\
$\begin{array}{l}\text { Bilateral sequelae of pleurisy } \\
\text { Asbestosis (with pleural plaques }\end{array}$ & 48 & $3 \cdot 6$ \\
$\begin{array}{l}\text { only) } \\
\text { Asbestosis with unilateral sequelae } \\
\text { of pleurisy }\end{array}$ & 29 & $2 \cdot 2$ \\
$\begin{array}{l}\text { Asbestosis with bilateral } \\
\text { sequelae of pleurisy }\end{array}$ & 9 & 0.7 \\
Total & 23 & $1 \cdot 7$ \\
\hline
\end{tabular}

\section{Discussion}

Pleural lesions due to exposure to asbestos are common in most industrialised societies. The county of Uppsala has few heavy industries and there are no asbestos factories, shipyards, or asbestos deposits. Despite this, at least $1-2 \%$ of the men above age 40 show typical pleural plaques on their chest radiographs. ${ }^{12}$ Most of these plaque carriers are construction workers, plumbers, electricians, and the like, with not very heavy and usually intermittent exposure to asbestos three or more decades ago. Asbestos pleurisy is not rare, although it is much less common than pleural plaques. ${ }^{10}$

Most of the many papers on asbestos related changes do not specify the pleural lesions. The ILO classification, though useful for parenchymal fibrosis, is of little assistance in distinguishing these changes. Following the gradual development of such lesions in more than 1000 people for 15-20 years has gradually led to the classification of intrathoracic asbestos related changes into three main varieties: (1) parietal pleural plaques, with slow progression over decades and never any sudden changes; (2) "classical" asbestosis-that is, diffuse parenchymal fibrosis, mainly in the lower lobes of the lung, which like the plaques gradually progresses without sudden deterioration; and (3) the acute pleurisy, which 
Table 2 Erythrocyte sedimentation rate (ESR) in different types of asbestos related pleuropulmonary lesions

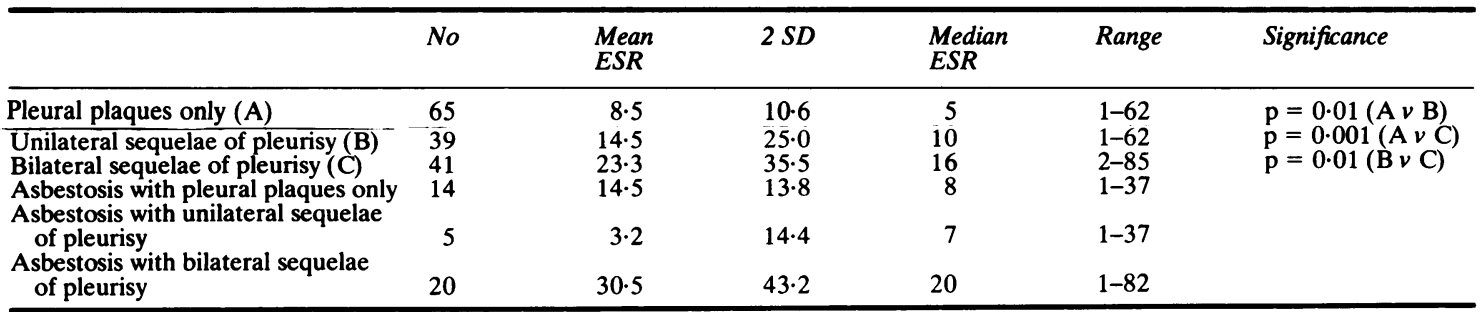

can either disappear without trace or leave blunted costophrenic angles, diffuse pleural thickening, pleuropulmonary fibrous strands ("crow's feet"), or rounded atelectasis (or any combination of these). Although the chest radiographic appearance of these last manifestations can be very different, the computed tomagraphy reveals their close association.

Computed tomography of pleural plaques shows a clear demarcation from the healthy lung (fig 1); in the patients with sequelae of pleurisy more or less pronounced fibrous strands penetrate into the lung parenchyma. Usually the classification from a plain chest radiograph is not difficult with some training. The definition of pleural plaques used in this study has a high specificity, to judge by necropsy findings." Unlike pleural plaques, the sequelae of pleurisy are not pathognomonic for asbestos exposure but can be seen after pleurisy due to other causes.

The ESR is a very non-specific test, and further investigations are necessary to establish the factors underlying the high values reported here. Asbestos in known to affect the immune system: the number and function of $\mathrm{T}$ lymphocytes are usually depressed $^{12-14}$ while the B cell function is enhanced, with increased concentrations of immunoglobulins and various autoantibodies. ${ }^{1315} 17$ The sudden appearance of asbestos pleurisy suggests that something has triggered an immune response; this could, for example, be a virus infection, since asbestos may depress cellular interferon induction ${ }^{18}$ and may thereby lower the defences against viruses.

The episodes of asbestos pleurisy associated with a raised ESR can last for months, sometimes recurring on the same or on the other side. These features are reminiscent of changes seen in rheumatoid arthritis, other autoimmune diseases, and certain drug reactions. Possibly, some kind of autoimmune response, triggered by asbestos, is concerned. Classical asbestosis and pleural plaques, which have a different natural history, may be caused by some other mechanism.

Closer attention should be paid in the future to the various types of pleural lesions caused by asbes- tos. The fact that asbestos pleurisy is more often than not symptomless ${ }^{10}$ is probably one of the reasons for its relative neglect so far. Interestingly, a recent study from Turkey ${ }^{19}$ indicates that in a population where sequelae of asbestos pleurisy are common the risk of developing mesothelioma is higher than in an exposed population where they are relatively less common, despite the fact that the prevalence of pleural plaques is about equal in the two communities. If the Turkish findings can be confirmed, and if the differences in ESR can be

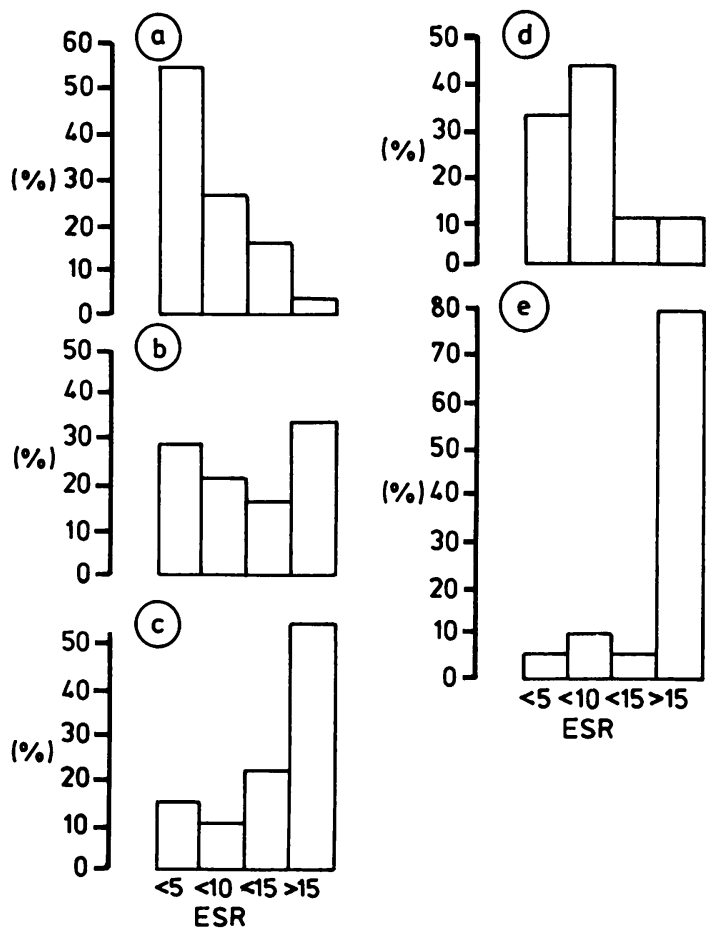

Fig 4 Erythrocyte sedimentation rate (ESR) associated with different types of asbestos related pleuropulmonary lesions. $a$-pleural plaques only $(N=65) ; b$-unilateral sequelae of pleurisy $(N=39) ; c$-bilateral sequelae of pleurisy $(N=41)$; $d$-asbestosis with pleural plaques only and with unilateral sequelae of pleurisy $(N=19)$; $e$-asbestosis with bilateral sequelae of pleurisy $(N=20)$. 
shown to be due to some effect on the immune system, this might shed some light on the pathogenesis of mesothelioma.

I wish to express my sincere thanks to Dr Anders Hemmingsson at the Department of Clinical Radiology for his invaluable help in providing the computed tomography scan pictures.

\section{References}

${ }^{1}$ Hillerdal G, Hillerdal O, Nöu E. Radiologically visible pleural plaques in a one year material from a health survey in 1976. Eur $J$ Respir Dis 1980;61,suppl 107:89-98.

${ }^{2}$ Hillerdal G. Pleural plaques in a health survey material. Frequency, development, and exposure to asbestos. Eur J Respir Dis 1978;59:257-63.

${ }^{3}$ Mackenzie FAF, Harries PG. Changing attitudes to the diagnosis of asbestos disease. J $R$ Nav Med Serv 1970;56:116-23.

${ }^{4}$ Hanke R. Rundatelektasen. Frotschr Röntgenstr 1971;114: 164-83.

${ }^{5}$ Hillerdal G, Hemmingsson A. Pulmonary pseudotumours and asbestos. Acta Radiol 1980;21:615-20.

${ }^{6}$ Mintzer RA, Cugell DW. The association of asbestosinduced pleural disease and rounded atelectasis. Chest 1982;81:457-60.

' Statistical Analysis Systems Institute. SAS users' guide: statistics. 1982 ed. Cary, North Carolina: SAS Institute, 1983.

${ }^{8}$ Bergström R. Different methods for variation analysis in unbalanced situations [Swedish]. Uppsala: Institute of Statistics, 1979.

${ }^{9}$ Searle SR. Linear models. New York: J Wiley and Sons, 1971.

${ }^{10}$ Hillerdal G. Non-malignant asbestos pleural disease. Thorax 1981;36:669-75.

" Hillerdal G, Lindgren A. Pleural plaques: correlation of autopsy findings to radiographic findings and occupational history. Eur J Respir Dis 1980;61:315-9.

12 Kang KY, Sera Y, Okochi T, Yamamura Y. T lymphocytes in asbestosis. $N$ Engl J Med 1974;291:735-6.

${ }^{13}$ Kagan E, Solomon A, Cochrane JG, Kuba P, Rocks PH, Webster I. Immunological studies of patients with asbestosis. II. Studies of circulating lymphoid cell numbers and humoral immunity. Clin Exp Immunol 1977;28:268-75.

${ }^{14}$ Campbell MJ, Wagner MMF, Scott MP, Brown DG. Sequential immunological studies in an asbestosexposed population. II. Factors affecting lymphocyte function. Clin Exp Immunol 1980;39:176-82.

is Turner-Warwick M, Parkes WR. Circulating rheumatoid and antinuclear factors in asbestos workers. $\mathrm{Br} \mathrm{Med} \mathrm{J}$ 1970; iii:492-5.

${ }^{16}$ Huuskonen MS, Räsäonen JA, Härkönen H, Asp S. Asbestos exposure as a cause of immunological stimulation. Scand J Respir Dis 1978;59:326-32.

${ }^{17}$ Lange A. An epidemiological survey of immunological abnormalities in asbestos workers. I-Non-organ and organ-specific autoantibodies. Environ Res 1980;22:162-75.

${ }^{18}$ Hahon N, Eckert HL. Depression of viral interferon induction in cell monolayers by asbestos fibers. Environ Res 1976;11:52-6.

${ }^{19}$ Hillerdal G, Baris YI. Radiological study of pleural changes in relation to mesothelioma in Turkey. Thorax 1983;38:443-8. 